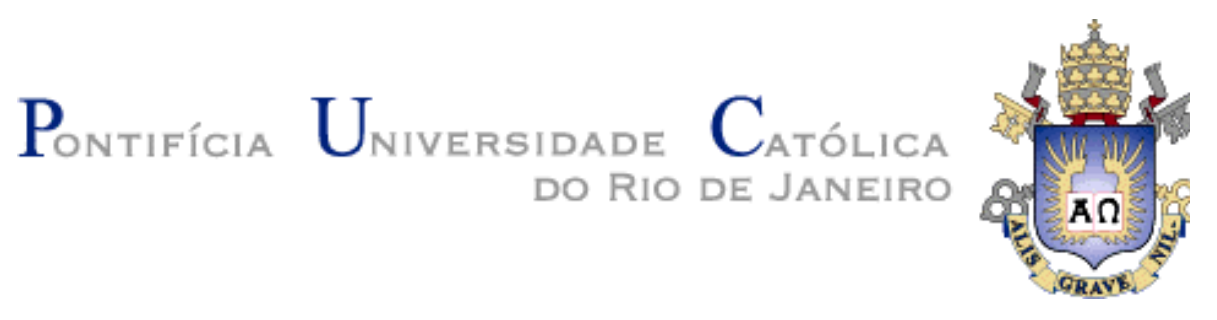

Roberta de Souza Coelho

\title{
Analyzing Exception Flows of Aspect-Oriented Programs
}

\section{PhD Thesis}

Dissertation presented to Pontifícia Universidade Católica do Rio de Janeiro, Departamento de Informática as part of the requirements for the fulfillment of the doctor's degree in Computer Science.

Supervisor: Arndt von Staa

Co-supervisor: Awais Rashid

Rio de Janeiro

June, 2008 


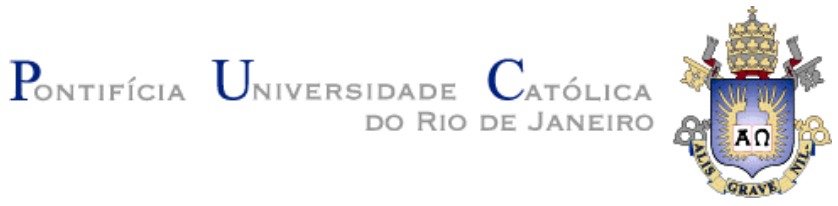

Roberta de Souza Coelho

\section{Analyzing Exception Flows of Aspect-Oriented Programs}

Thesis presented in the Graduate Program in Computer Science of the Pontifícia Universidade Católica do Rio de Janeiro in partial fulfillment of the requirements for the degree of Doctor in Computer Science. Approved by the following Examination Committee.

Prof. Arndt Von Staa

Orientador

Departamento de Informática - PUC-Rio

Prof. José Carlos Pereira de Lucena

Departamento de Informática - PUC-Rio

Prof. Renato Fontoura de Gusmão Cerqueira

Departamento de Artes e Design - PUC-Rio

Prof. Paulo Henrique Monteiro Borba Centro de Informática - UFPE

Prof. Paulo Cesar Masiero

Instituto de Ciências Matemáticas e de Computação - USP

Prof. José Eugenio Leal

Graduate Programs' Coordinator for the Center of Science and Techonology -

PUC-Rio 
Todos os direitos reservados. É proibida a reprodução total ou parcial do trabalho sem autorização da universidade, da autora e dos orientadores.

\section{Roberta de Souza Coelho}

Graduou-se no Curso de Bacharelado em Ciência da Computação da Universidade Federal de Campina Grande (UFCG) em 1999. Obteve o título de Mestre em Ciência da Computação na Universidade Federal de Pernambuco (UFPE) em 2002. Atuou como coordenadora de projetos, engenheira de software no Centro de Estudos e Sistemas Avançados do Recife (CESAR) de 1999 a 2003. Ainda em 2003 atuou como pesquisadora no projeto Ourgrid/UFCGHP. Atuou como pesquisadora na área de Desenvolvimento de Software Orientado a Aspectos e Teste de Software no Laboratório de Engenharia de Software (LES/PUC-Rio) de 2004 a 2007, e no Tecgraf/PUC-Rio em 2006.

Ficha Catalográfica

Coelho, Roberta de Souza

Analyzing exception flows of aspect-oriented programs / Roberta de Souza Coelho ; supervisor: Arndt Von Staa; co-supervisor: Awais Rashid. 2008.

173 f. ; $30 \mathrm{~cm}$

Tese (Doutorado em Informática)-Pontifícia Universidade Católica do Rio de Janeiro, Rio de Janeiro, 2008.

Inclui bibliografia

1. Informática - Teses. 2. Tratamento de exceções. 3. Programas orientados a aspectos. 4. Análise estática. 5. Estudo empírico. 6. Exceções não capturadas. 7. Padrões de erro. I. I. Staa, Arndt von. II. Raschid, Awais. III. Pontifícia Universidade Católica do Rio de Janeiro. Departamento de Informática. IV. Título. 
A Deus por sempre estar presente em minha vida através do amor da minha família e amigos.

To God for always being present in my life through the love of my family and friends. 


\section{Acknowledgment}

First, I give thanks to God for giving me the greatest gift of my life: my beloved family. To my dear parents Fatima and Roberto for loving and supporting me since I was born. Their love has always played a fundamental role on the achievements of my

life. Even living several kilometers apart during my $\mathrm{PhD}$, they were always very present! To my husband Uirá for his love and for supporting me along the $\mathrm{PhD}$ journey. God helped us to overcome the difficulties inherent to $\mathrm{PhDs}-$ he was also doing his $\mathrm{PhD}$ at the same time ;-). To my beloved sister Renata (Nini) and dearest brother Jaime (Jaimão) for their love, care and words of wisdom. I love them so much!

To my advisor, Arndt von Staa who was always very supportive, and whose "investigative spirit" was a great inspiration to this $\mathrm{PhD}$ work. Our discussions about the real pros and cons of Aspects were the seeds of this thesis. Besides inspiring my $\mathrm{PhD}$ work, this same spirit also inspired other great empirical studies carried out by the Software Engineering Laboratory (LES).

To Professor Awais Rashid for the great discussions on AO development and for advising me during the seven-month exchange period at Lancaster University.

To Professor Carlos Lucena for his support and for creating opportunities of research collaboration.

To my friend and PhD colleague Fabiano Ferrari who contributed on the empirical study performed in this work - helping me to manually inspect the exception handling code of several system versions. To my friend and $\mathrm{PhD}$ colleague Cláudio (Baiano) who also contributed with great discussions. To my friend Elder Cirilo who implemented one of the tools developed during the $\mathrm{PhD}$, and to the LES research group for the discussions over those years (on Friday's Seminars). To my dear husband and $\mathrm{PhD}$ colleague Uirá for our interesting technical discussions about aspects and testing.

To my dearest grandmother, Lilia, for always loving and motivating me. She is always very lively and funny, and often called me to say: "Dear Robertinha, you should finish your studies and come back home. I miss you. You have studied too much already ;-)!". She was always a great motivation to me.

To Maité (Teté) and Nandinho for opening not only the doors of their home but also to the doors of their hearts, and enabling us to live great moments together with them and my dear nephew Cauê. To D. Tereza e S. Tek for their care and attention.

To my friends from Centro Loyola de Fé e Cultura and Centro de Pastoral 
Anchieta da PUC-Rio, especially to: my dearest friend Fernanda Motta (Fê), who shared with me words of wisdom and her friendship; Helena, for being so peaceful and friendly; and Pe Emmanuel, a blessed friend, who is a real image of Christ in our days.

To all my friends from Chaplaincy Center at Lancaster University specially for Pr. Hugh, Sister Ella, Jim, Rosa, Veronica, Robert, Kersting, Gosia and Mariel. They represented real gifts from God while I was in Lancaster (The Friendship Icon)

To my friends from Tecgraf: Julia, Leticia, Clarissa, Taciana, Malu, Cris, Ana Moura, Ana and Andrea. We formed a software engineering group composed mostly by women - which is very rare is computer science. It was a very special team! Really friendly and competent! My experience at Tecgraf was was one of the inspirations of my research on exception handling.

To my friends Silvia Passos, Mariza Lucena and Vera Menezes for their friendship and sympathy!

To my friends from the Northeast, who also came to Rio study at PUC, Silvinha, Suzana. We laugh a lot, and shared great moments and the same accent!

To my friends from DobrasBrasil: Sol (in memorium), a Amália, Jaciara e Glória. Their enthusiasm and happiness made the Saturday's afternoons very special.

To AOSD Research group of Lancaster University especially Alessandro and Nélio for their feedback on the work presented here and for the interesting discussions concerning exception handling.

To the professors of the committee, Paulo Borba, Renato Cerqueira e Paulo Masiero for giving great suggestions on how to improve this thesis.

To the Informatics Department, its professors and the technical staff, for supporting my research, and to CNPq, CAPES and FAPERJ for funding it. 


\section{Abstract}

Coelho, Roberta; von Staa, Arndt. Analyzing Exception Flows of Aspect-Oriented Programs. Rio de Janeiro, 2008. 173p. PhD Thesis Departamento de Informática, Pontifícia Universidade Católica do Rio de Janeiro.

It has been empirically observed that aspect-oriented (AO) decompositions promote the modularity and the design stability of software systems containing crosscutting concerns. However, most of this existing empirical research has focused on the positive and negative impacts of $\mathrm{AO}$ programming in the modularization of crosscutting concerns in the context of "normal" control flow of programs. Consequently, most of these works do not account for the exceptions that may flow from aspects: when an aspect adds a new functionality to specific points in the code, this additional behavior may also bring new exceptions. An aspect also has the ability to handle exceptions that were previously handled inside the base code. Moreover, the exceptions that escape form aspects also flow inside the program, and may lead to unexpected error-prone scenarios, such as: unintended handler actions and uncaught exceptions. This thesis presents an empirical study to evaluate the impact of aspects on the exception flow of programs. To support the reasoning about the flow of exceptions on AO programs, a static analysis tool called SAFE (Static Analysis for the Flow of Exceptions) was implemented. Based on data empirically collected during the study we characterized a catalogue of bug patterns related to the exception handling code of AO programs. To help AO developers to check the reliability of the exception handling code, this work presents verification approach - supported by the SAFE tool - which provides guidelines to counter these bug patterns. Our findings show that the exception handling code in aspect-oriented programs tends to be error-prone, but that a verification approach based on static analysis can lead to significant improvements.

\section{Keywords}

Exception handling, aspect-oriented programs, static analysis, empirical study, uncaught exceptions, bug patterns. 


\section{Resumo}

Coelho, Roberta; von Staa, Arndt. Analisando o Fluxo de Exceções em Programas orientados a Aspectos. Rio de Janeiro, 2008. 173p. Tese de Doutorado - Departamento de Informática, Pontifícia Universidade Católica do Rio de Janeiro.

Os mecanismos de tratamento de exceções têm o objetivo de aumentar a robustez e a modularidade do software na medida em que promovem a separação entre o código dedicado ao tratamento de erros e código associado ao fluxo normal de execução do programa. Estes permitem a detecção de erros e a associação de respostas adequadas aos mesmos, através da execução de códigos de recuperação que são geralmente encapsulados em tratadores (do inglês: handlers). A importância dos mecanismos de tratamento de exceção é atestada pelo fato destes fazerem parte da maioria das linguagens de programação mais utilizadas na atualidade: Java, C++ e C\#.

$\mathrm{Na}$ última década a programação orientada a aspectos (POA) vem sendo amplamente utilizada como forma de modularizar interesses que se encontram espalhados nas decomposições primarias de um sistema (e.g., funções, classes, métodos) em uma abstração chamada aspecto. Os aspectos utilizam construções especificas para promover modificações externas nos programas, incluindo comportamentos adicionais em pontos específicos do código. POA vem sido utilizado com o objetivo de aumentar a modularidade do código de tratamento de exceção e de interesses transversais igualmente importantes tais como: persistência, distribuição, segurança, controle de transações e monitoramento.

De acordo com alguns estudos empíricos, POA tem sido usado com sucesso com o objetivo de promover o tratamento de erro modular em vários cenários de tratamento de exceções. Porém, é sabido que mecanismos de programação flexíveis (e.g., herança e polimorfismo) podem ter um efeito negativo no tratamento de exceções. Se por um lado os mecanismos de composição POA baseados na inversão de controle podem trazer um novo leque de possibilidades de projeto, promovendo em muitas circunstâncias uma maior estabilidade do projeto (do inglês: design stability), eles podem perder seu valor se ele torna o tratamento de exceções propenso a erros (do inglês: error-prone). Refinamento aspectuais do comportamento do código base, podem por um lado promover a robustez do sistema em situações onde exceções são lançadas ou contribuir para os problemas típicos de tratamento de exceções mal projetados tais como: (i) exceções não capturadas (do inglês: uncaught exceptions); (ii) exceções 
capturadas por tratadores genéricos (do inglês: exception subsumption) (i.e., exceções capturadas por um tratador cujo argumento é um supertipo da exceção a ser tratada); (iii) e exceções capturadas por tratadores errados (do inglês: unintended handler action).

Infelizmente, não existe nenhuma avaliação sistemática dos efeitos positivos e negativos da programação orientada a aspectos na robustez do código de tratamento de exceções. Os trabalhos de pesquisa descritos na literatura têm se limitado a analisar os impactos dos aspectos no fluxo normal de execução do programa. Além disso, a maioria dos estudos empíricos não vai além de discussões sobre os ganhos de modularidade e as ciladas associadas à utilização de aspectos - para o tratamento de exceções e de outros interesses transversais. Por exemplo, estes estudos não levam em consideração as conseqüências inerentes as novas exceções e tratadores que são adicionadas ao código base junto com a nova funcionalidade adicionada pelos aspectos.

Esta tese descreve o primeiro estudo sistemático que avalia quantitativamente o impacto da composição aspectual no fluxo excepcional de programas. Este estudo se baseou na utilização de uma ferramenta de análise do fluxo de exceções chamada SAFE (do inglês: Static Analysis for the Flow of Exceptions) desenvolvida ao longo deste trabalho e na inspeção manual do código de tratamento de exceções de um conjunto de sistemas para os quais a versão Java e AspectJ estavam disponíveis. A ferramenta SAFE foi construída com base no framework SOOT para a análise e otimização de bytecode. A partir de uma representação do programa construída a partir do bytecode do código base combinado com os aspectos (do inglês: woven bytecode), a ferramenta calcula estaticamente os caminhos excepcionais do programa (i.e., caminhos no grafo de chamadas que ligam o elemento que lança uma exceção ao elemento que contém o tratador que irá capturá-la). Estes caminhos são então armazenados servindo como base para as atividades de inspeção manual do código de tratamento de exceções.

Três sistemas de médio porte foram avaliados neste estudo: Health Watcher um sistema Web que permite o registro de queixas para sistema público de saúde; o Mobile Photo - uma linha de produto de software para aplicações de manipulação de media (arquivos de som, imagem e vídeo) em dispositivos móveis; e o JHotDraw - um framework open source para construção de aplicações de edição gráfica. Para os dois primeiros sistemas mais de release foi avaliado. Ao todo 10 versões foram avaliadas, totalizando $41.1 \mathrm{KLOC}$ de código Java dos quais aproximadamente 4.1 KLOC eram dedicados ao 
tratamento de exceções, e 39 KLOC linhas de código AspectJ dos quais aproximadamente 3.2 KLOC eram dedicadas ao tratamento de exceções.

Alem de representarem aplicações de diferentes domínios e as aplicações analisadas neste estudo possuem estratégias de tratamento de exceções diferentes. No Health Watcher apenas o código de tratamento de exceções das funcionalidades transversais (representadas por aspectos) foram movidas para aspectos de tratamento de exceções (Exception Handling Aspects). No Mobile Photo a maioria do código de tratamento de exceções foi aspectualizado - não apenas o tratamento de exceções dos interesses transversais. Já no JHotDraw, o código de tratamento de exceções da versão AspectJ permaneceu no código base.

Alguns resultados negativos foram detectados de forma consistente através da análise das versões AO de cada sistema, quais sejam: (i) um maior número de caminhos excepcionais que continham exceções não capturadas (do inglês: uncaught exceptions) foi encontrado nas versões que usavam aspectos como tratadores de exceções - exceções não capturadas podem levar a crashs no programa de forma imprevisível; e (i) um maior número de exceções capturadas por subsumption algumas delas levando ao tratamento inadequado de exceções, i.e, exceções lançadas por aspectos e capturadas erroneamente por tratadores presentes no código base.

Neste trabalho investigamos as causas para tais aumentos e apresentamos as mesmas na forma de um catálogo de padrões de erro (do inglês: bug pattern) relacionado ao código de tratamento de exceções de programas AspectJ. Além disso, este trabalho propõe uma abordagem de verificação, baseada na utilização da ferramenta SAFE, com o objetivo de auxiliar os desenvolvedores na detecção e solução de potenciais faltas no código de tratamento de exceções de sistemas AO.

São, portanto, contribuições deste trabalho: (i) a realização da primeira análise sistemática com o objetivo de investigar como os aspectos afetam o fluxo excepcional dos programas; (ii) a construção de uma ferramenta de análise estática do fluxo excepcional de sistemas Java e AspectJ; (iii) a definição de um catálogo padrões de erro associado ao código de tratamento de exceções de sistemas AspectJ, o qual foi obtido através de dados empiricamente coletados; e (iv) a definição e validação inicial de uma abordagem de verificação, baseada na utilização da ferramenta SAFE para detecção e solução de potenciais faltas 
no código de tratamento de exceções de sistemas AO.

Os resultados deste trabalho mostraram que o código de tratamento de exceções de programas orientados a aspectos é, de fato, propenso a falhas; mas uma abordagem de verificação baseada em analise estática pode reduzir significativamente o grau em que estas falhas acontecem. As contribuições alcançadas neste trabalho permitirão que: (i) desenvolvedores de aplicações AO avaliem o impacto causado pelos aspectos no fluxo excepcional do programa ao refatorar ou implementar um sistema AO; e (ii) projetistas de linguagens $\mathrm{AO}$ considerem a realização de refinamentos nos mecanismos de tratamento de exceções existentes, de forma a torná-los mais robustos e resilientes aos tipos de erros encontrados neste estudo.

\section{Palavras-chave}

Tratamento de exceções, programas orientados a aspectos, análise estática, estudo empírico, exceções não capturadas, padrões de erro. 


\section{Index}

1 Introduction 20

1.1. The Problem 21

1.2. Summary of Goals 22

1.3. Thesis Structure 23

2 Background $\quad 25$

2.1. Aspect-Oriented Programming 25

2.1.1. Aspect Weaving 25

2.1.2. Obliviousness and Quantification Properties 26

2.1.3. Crosscutting Interfaces (XPI) 27

2.1.4. Aspect J 28

2.1.4.1. AspectJ Main Constructs $\quad 29$

2.1.5. Other AO Languages 31

2.2. Exception Handling Mechanisms 32

2.2.1. Exception Handling Mechanism in AspectJ Programs 33

2.2.2. Exception Handling Constructs in AspectJ 36

2.3. Checking the Reliability of Exception Handling Code 39

2.3.1. Checking the Reliability through Testing 39

2.3.2. Checking the Reliability through Static Analysis 40

2.4. Summary 41

3 Characterizing the Exception Flow in Aspect-Oriented Programs: The Empirical Study 43

3.1. Study Setting 43

3.1.1. Target Systems 44

3.1.1.1. Health Watcher 44

3.1.1.2. Mobile Photo 46

3.1.1.3. JHotDraw 48

3.1.1.4. Characteristics of the Target Systems 50 
3.1.2. Reasoning About the Exceptional Behavior 52

3.1.3. Automated Exception Flow Analysis 52

3.1.4. Inspection of Exception Handlers 53

3.1.5. Study Operation $\quad 55$

3.2. Data Analysis and Interpretation 56

3.2.1. Empirical Data $\quad 57$

3.2.1.1. The Impact of Aspects on How Exceptions are Handled 58

3.2.1.2. The Blame for Uncaught Exceptions and Subsumptions $\quad 59$

3.2.1.3. Are All Exceptions Signaled by Aspects becoming Uncaught or Caught by Subsumption? 61

3.2.2. Detailed Inspection 61

3.2.2.1. Health Watcher 63

3.2.2.2. Mobile Photo 64

3.2.2.3. JHotDraw 64

3.2.3. Study Constraints 64

3.3. Summary 65

4 The Empirical Study Results $\quad 67$

4.1. Bug Patterns in the Exception Handling Code of AO Systems 67

4.1.1. Aspects as Exception Handlers 68

4.1.2. Aspects as Exception Signalers 71

4.1.3. Softening Exceptions 73

4.2. Discussions and Study Constraints 75

4.2.1. Representativeness 76

4.2.2. Exception Handling vs. AOP Properties 78

4.2.3. Additional Lessons Learned 79

4.3. Summary 81

5 Exception-Flow Analysis for AspectJ Programs 82

5.1. Supporting Ideas 82

5.1.1. Advice Weaving in AspectJ 83

5.1.2. Program Representation $\quad 87$

5.1.2.1. Dealing with Dynamic Dispatch 89

5.2. Heuristics used by the Tool 89 
5.2.1. Blame Assignment in Exception Handling Scenarios 89

5.2.2. Exception Paths in AO Systems 91

5.2.2.1. Exception Paths Originating from Exception Softening 92

5.2.2.2. Exception Paths Originating from Library Methods 93

5.3. The SAFE Tool 94

5.3.1. The Tool's Architecture 94

5.3.2. The Exception Analysis Component 96

5.3.2.1. Integration with Soot Framework 98

5.3.3. The Call Graph Builder 100

5.3.4. The Exception Path Miner 102

5.4. Implementation Details 104

5.4.1. Soot Intermediate Representation 105

5.4.2. Exceptional Control Flow 106

5.4.3. Exception Filtering 108

5.4.4. Dealing with Exception-Related AspectJ Weaver Residues 109

5.5. Tool's Performance 111

5.6. Summary 116

6 The Approach $\quad 118$

6.1. Checking the Reliability of Exception Handling Code 118

6.1.1. The Characteristics of $A O$ Compositions $x$ the Development of Exception-ware AO Systems 121

6.2. Status of Current Aspect Libraries 124

6.3. The Verification Approach 125

6.4. Worked Example 129

6.4.1. Discover the Exception Interfaces of Aspects 130

6.4.2. Specify the Exception Handling Contracts 134

6.4.3. Implement Exception Handling Code 136

6.4.4. Calculate Exception Paths 137

6.4.5. Manually Inspect the Exception Handling Code 138

6.5. The Approach's Effectiveness x the SAFE Tool's Precision 139

6.6. Discussions and Lessons Learned 142

6.7. Summary 144 
7 Related Work 146

7.1. Static Analysis Tools and Techniques 146

7.2. Empirical Studies regarding the Exception Handling Code 148

7.3. AO Fault Models and Bug Patterns 150

7.4. Aspect Interactions 151

7.5. Verification Approaches for AO Systems 153

7.6. Collateral Effects of Aspect Libraries Reuse 154

$\begin{array}{ll}\text { 7.7. Summary } & 155\end{array}$

8 Concluding Remarks and Future Work 156

8.1. Conclusions 156

8.2. Contributions 158

8.3. Future Work 159

$\begin{array}{ll}\text { 8.4. PhD Roadmap } & 161\end{array}$

9 References 165 


\section{List of Figures}

Figure 1. Exception-aware method call chain in AO programs...............................34

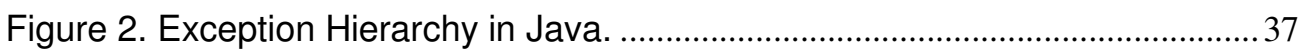

Figure 3. The $\mathrm{OO}$ design of Health Watcher system (version 9) ...........................4 45

Figure 4. The AO design of Health Watcher system (version 9). ...........................46

Figure 5. The $\mathrm{OO}$ design of Mobile Photo System (version 6)................................47

Figure 6. The AO design of Mobile Photo System (version 6) ............................... 48

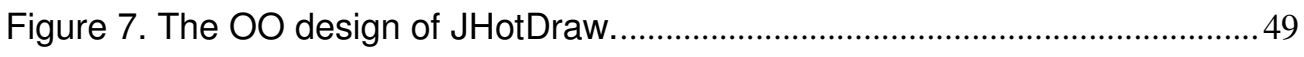

Figure 8. The AO design of AJHotDraw. .................................................................. 49

Figure 9. Each step conducted in the study operation. ..........................................55

Figure 10. Uncaught exceptions, subsumptions, and specialized handlers per

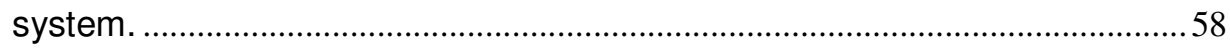

Figure 11. Percentage of uncaught exceptions, subsumptions, and specialized

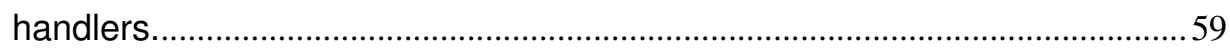

Figure 12. Handler type of exceptions thrown by aspects. ..................................... 61

Figure 13. The handler action per target system....................................................63

Figure 14. Schematic view of the Late Binding Handler. ...................................... 70

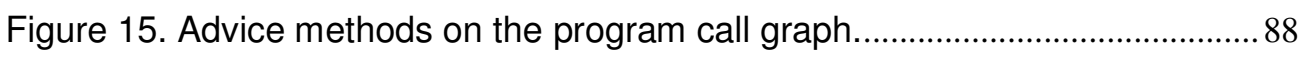

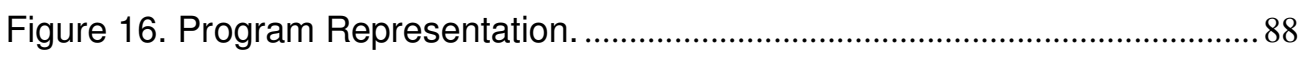

Figure 17. Scenarios where advice act as exception signalers. ............................90

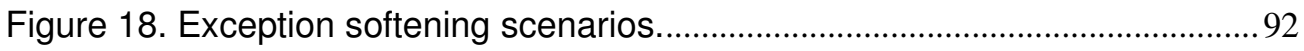

Figure 19. The exception types and the exception interface of a method.............93

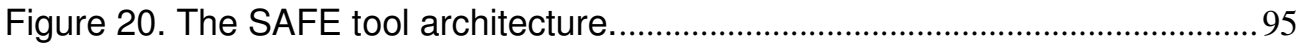

Figure 21. Three-dimension classification for exception paths. ........................... 103

Figure 22. Call graph vertex internal structure ..................................................... 108

Figure 23. Library methods in an exception path. ................................................115 
Figure 24. Types of aspects in an $\mathrm{AO}$ application...............................................119

Figure 25. Consequences of invasiveness modifications ..................................... 122

Figure 26. Consequences of quantification property in the presence of

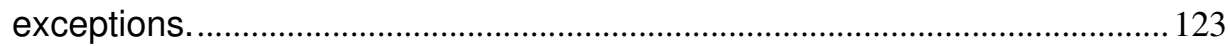

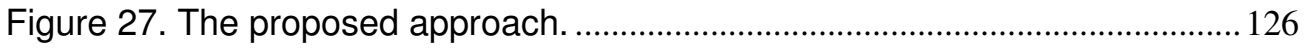

Figure 28. The extended architecture of Health Watcher system.........................130

Figure 29. Research works organized in a timeline ............................................ 162 


\section{List of Tables}

Table 1. Main pointcut designators of AspectJ language........................................... 30

Table 2. Crosscutting concerns per target system version. ...................................50

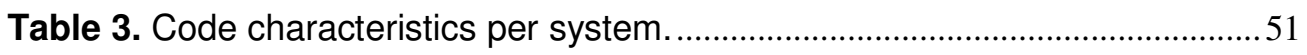

Table 4. Categories of handler actions and corresponding descriptions ..............54

Table 5. Classification of exception paths per target system................................57

Table 6. Classification of exception paths according to handler actions...............62 62

Table 7. Distribution of the bug patterns per system.............................................6

Table 8. AO languages characteristics: advice types and exceptions that may

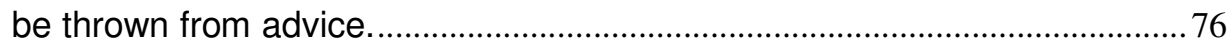

Table 9. Differences on available pointcut designators. .......................................... 77

Table 10. Representation of aspect advice on Java bytecode. ............................. 86

Table 11. The exception analysis performance using the SAFE tool..................112

Table 12. Characteristics of the exception handling $(\mathrm{EH})$ code on aspect libraries.

Table 13. Exception paths calculated by SAFE tool.............................................. 134

Table 14. Reported and spurious exception paths............................................ 140

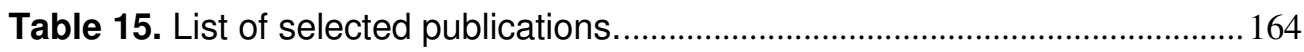




\section{List of Abbreviations}

$\begin{array}{ll}\text { AOSD } & \text { Aspect Oriented Software Development } \\ \text { AOP } & \text { Aspect Oriented Programming } \\ \text { AO } & \text { Aspect Oriented } \\ \text { OO } & \text { Object Oriented } \\ \text { GUI } & \text { Graphical User Interface } \\ \text { XPI } & \text { Crosscutting Interface } \\ \text { HW } & \text { Health Watcher system } \\ \text { MP } & \text { Mobile Photo system } \\ \text { JHD } & \text { JHotDraw system }\end{array}$

